\title{
Ultrasound Imaging Algorithm: Half-Matrix Focusing Method Based on Reciprocity
}

\author{
Xufei Guo ${ }^{D}$, ${ }^{1,2}$ Yan Han, ${ }^{1}$ and Pengfei Nie $^{1}$ \\ ${ }^{1}$ Shanxi Key Laboratory of Signal Capturing \& Processing, North University of China, Taiyuan 030051, China \\ ${ }^{2}$ Lvliang University, Lvliang 033000, China \\ Correspondence should be addressed to Xufei Guo; gx_nuc@163.com
}

Received 30 September 2020; Revised 11 January 2021; Accepted 16 January 2021; Published 2 February 2021

Academic Editor: Thomas Schuster

Copyright (c) 2021 Xufei Guo et al. This is an open access article distributed under the Creative Commons Attribution License, which permits unrestricted use, distribution, and reproduction in any medium, provided the original work is properly cited.

The ultrasonic phased array total focusing method (TFM) has the advantages of high imaging signal-to-noise ratio (SNR) and high defect resolution, but the problem of large amount data capturing and processing limits its practical industrial applications. To reduce the imaging calculation demand of the total focusing method, a half-matrix focusing method (HFM) is proposed based on the acoustic reciprocity. The method simplifies the calculation process of full-matrix data capturing (FMC) and total focus imaging. The experimental results show that the signal obtained by the linear array transceiver sensor is highly consistent, and the imaging resolution and signal-to-noise ratio of the half-matrix focusing method are slightly lower than those of full-matrix focusing method and higher than those of the B-scan imaging. However, compared with TFM, data acquisition and computational efficiency using the HFM have been improved significantly.

\section{Introduction}

Ultrasonic phased array testing adopts multichannel delay transceiver technology to emit each array element so as to achieve the angle deflection of the acoustic beam and focus on depth changes and electronic scanning, which improve the ability to detect defects [1-4]. The ultrasonic phased array imaging method based on postprocessing has been deeply studied by scholars. Holmes et al. [5] first proposed the concepts of full-matrix capture (FMC) and established the full-focus imaging method (TFM) based on FMC. Compared with the conventional ultrasonic phased array focusing algorithm, TFM can realize the synthetic focusing of any point in the detection region and the imaging quality is obviously better [6-9]. This method has therefore been extensively used in the fields of aviation, nuclear power, human organs, and so on [10-12].

However, due to the large amount of full-matrix data involved, TFM imaging calculations are time-consuming and have poor real-time processing in real time, which limit their industrial applications. To improve imaging efficiency of TFM, Sutcliffe et al. [13] proposed a method of hardware acceleration of postprocessing using GPU. Bouaziz et al. $[14,15]$ proposed a real-time method that uses parallel computing with multiple field-programmable gate arrays. But, the cost will increase significantly by improving the hardware architecture. Another research goal is to improve the imaging algorithm while maintaining the image quality. Hunter et al. [16] introduced a generalization of the wavenumber algorithm for Fourier-domain full-matrix imaging. Hu et al. [17] proposed a sparse-TFM imaging method based on sparse array optimization and new edge-directed interpolation. For the problem of limited acquisition speed, Moreau et al. [18] proposed an effective aperture method based on far-field approximation. In order to improve the imaging efficiency, Zhao et al. [21] used the upper triangle data to complete the ultrasound focus imaging.

In the article, a half-matrix focusing method (HFM) based on transceiver sensor reciprocity is developed to improve inspection efficiency. This method can effectively 
reduce full-matrix data capturing and TFM imaging time while maintaining the image quality. The remainder of this paper is organized as follows: In Section 2, the FMC theory and the imaging methods are introduced. Section 3 presents the experimental methods and procedures for defect detection. Moreover, the experimental results of data processed with conventional TFM and HFM are analyzed. Section 4 compares HFM imaging and B-scan imaging and discusses the subsequent research directions of the HFM imaging. Finally, the conclusions are given in Section 5.

\section{Methods}

2.1. FMC and TFM. Figure 1 shows the principle of fullmatrix ultrasonic data capture $[19,20]$. A linear array consisting of $N$ elements excites the ultrasonic wave in sequence. During each excitation, the ultrasonic signal is received by every array element. Finally, an $N \times N$ A-scan data matrix is obtained. Here, $A_{\mathrm{ij}}(t)$ is the $N \times N$ A-scan signal emitted by element $i$ and received by element $j$. The format of full-matrix data is shown as follows:

$$
A=\left[\begin{array}{cccccc}
A_{11} & A_{12} & \ldots & A_{1 j} & \ldots & A_{1 N} \\
A_{21} & A_{22} & \ldots & A_{2 j} & \ldots & A_{2 N} \\
\ldots & \ldots & \ldots & \ldots & \ldots & \ldots \\
A_{i j} & A_{i 2} & \ldots & A_{i j} & \ldots & A_{i N} \\
\ldots & \ldots & \ldots & \ldots & \ldots & \ldots \\
A_{N 1} & A_{N 2} & \ldots & A_{N j} & \ldots & A_{N N}
\end{array}\right] .
$$

Figure 2 shows the principle of total focus algorithm $[19,20]$. Image reconstruction using full-matrix data is divided into two steps. First, discretization of the inspected region into a grid is performed. Secondly, calculation of the amplitude intensity at a grid point $P\left(x_{p}, z_{p}\right)$ is calculated by

$$
I_{p}\left(x_{p}, z_{p}\right)=\sum_{i=1}^{N} \sum_{j=1}^{N} A_{i j}\left(t_{i j}\left(x_{p}, z_{p}\right)\right)
$$

where $t_{i j}\left(x_{p}, z_{p}\right)$ is the time of flight starting from the transmitter element $i$, passing through the focusing point $P\left(x_{p}, z_{p}\right)$, and arriving at the receiver element $j$. The time of flight is defined by

$$
t_{i j}\left(x_{p}, z_{p}\right)=\frac{\left(\sqrt{\left(x_{i}-x_{p}\right)^{2}+z_{p}^{2}}+\sqrt{\left(x_{j}-x_{p}\right)^{2}+z_{p}^{2}}\right)}{c},
$$

where $c$ is the propagation velocity of ultrasound in the medium and $\left(x_{i}, 0\right)$ and $\left(x_{j}, 0\right)$ are the Cartesian coordinates of the transmitter and the receiver, respectively.

2.2. HFM. Consider that the performance indicators of each channel of the linear ultrasonic array transducer have excellent consistency. That is, the signal that is transmitted from element $i$ and received on element $j$ is identical to the signal transmitted on element $j$ and received on element $i$. It is named acoustic reciprocity. According to the principle of acoustic reciprocity, the full-matrix data shown in equation (1) have good symmetry. So the upper triangular or lower triangular matrix signal of the full-matrix data can be used in computational imaging. This method is called the half-matrix focus imaging (HFM), shown as follows (4) [21] or (5):

$$
\begin{aligned}
& I_{p}\left(x_{p}, z_{p}\right)=\sum_{i=1}^{N} \sum_{j=i}^{N} A_{i j}\left(t_{i j}\left(x_{p}, z_{p}\right)\right), \\
& I_{p}\left(x_{p}, z_{p}\right)=\sum_{i=j}^{N} \sum_{j=1}^{N} A_{i j}\left(t_{i j}\left(x_{p}, z_{p}\right)\right) .
\end{aligned}
$$

During data acquisition and imaging calculation, using the half-matrix focusing method, $N \times N$ sets of A-scan data will be reduced to $N \times((N+1) / 2)$. Before imaging calculation, it is necessary to normalize the image data. In the article, the amplitude data are normalized to between -128 and 127. It can superimpose and offset the random noise of positive and negative values [21]. The amplitude is normalized by

$$
\begin{aligned}
& I_{p}\left(x_{p}, z_{p}\right)=\frac{I_{p}\left(x_{p}, z_{p}\right)}{I_{\max }\left(x_{p}, z_{p}\right)} \times 127, \quad I_{p}\left(x_{p}, z_{p}\right) \geq 0, \\
& I_{p}\left(x_{p}, z_{p}\right)=-\frac{I_{p}\left(x_{p}, z_{p}\right)}{I_{\min }\left(x_{p}, z_{p}\right)} \times 128, \quad I_{p}\left(x_{p}, z_{p}\right)<0 .
\end{aligned}
$$

\section{Simulation and Results}

3.1. Experimental Conditions. The finite element method (FEM) was used to validate our proposed imaging algorithm, and ABAQUS 2016 (Dassault,Velizy-Villacoublay, France) software was used to simulate the full-matrix detection of defects in a Q235 steel plate. The finite element model is shown in Figure 3. The 32-element linear array transducer had a central frequency $\mathrm{f}=5 \mathrm{MHz}$ with an interelement spacing of $0.5 \mathrm{~mm}$ and an element width of $0.4 \mathrm{~mm}$. Fullarray probe parameters are listed in Table 1.

The defect positions in the steel plates are shown in Figure 4. The TFM and HFM were tested on three finite element models of isotropic steel plates $(100 \mathrm{~mm} \times 30 \mathrm{~mm})$. To suppress the interference of reflected waves, each model has infinite border with a thickness of $5 \mathrm{~mm}$. In model 4(a), there are three round through-hole defects (Nos. 1, 2, and 3) with diameters of $2 \mathrm{~mm}$. Between each defect, the lateral spacing was $5 \mathrm{~mm}$. The distance between defect No. 1 and the upper edge of the steel plate model was $14 \mathrm{~mm}$. In model 4(b), there is an oval through-hole (No. 4) with a long axis of $4 \mathrm{~mm}$ and a short axis of $2 \mathrm{~mm}$. The distance between the defect and the upper edge of the steel plate model was $19 \mathrm{~mm}$. In model 4(c), there is a rectangular through-hole (No. 5) defect with a length of $3 \mathrm{~mm}$ and width of $1 \mathrm{~mm}$. The 
$1 \sim \mathrm{N}$ array elements are sequentially excited

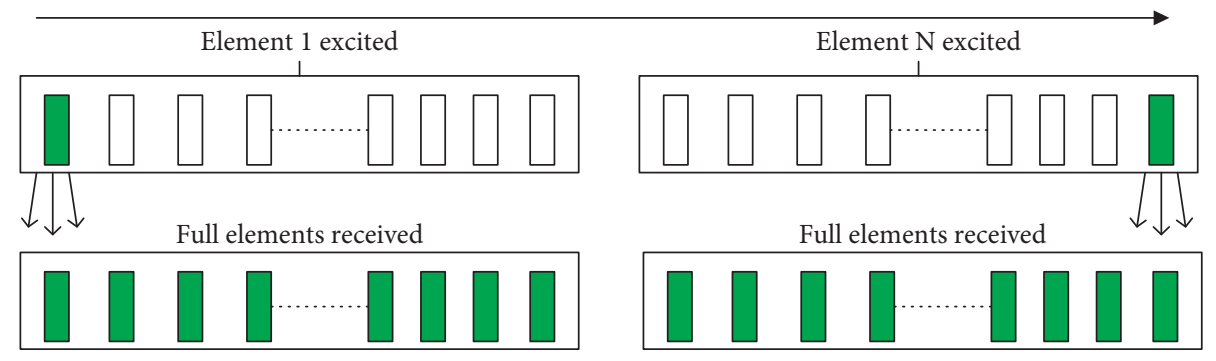

FIGURE 1: Schematic diagram of full-matrix data capture.

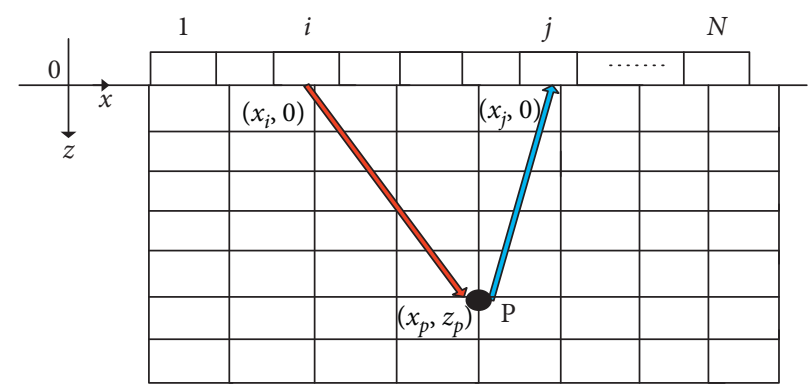

Figure 2: Schematic diagram of the total focus algorithm.

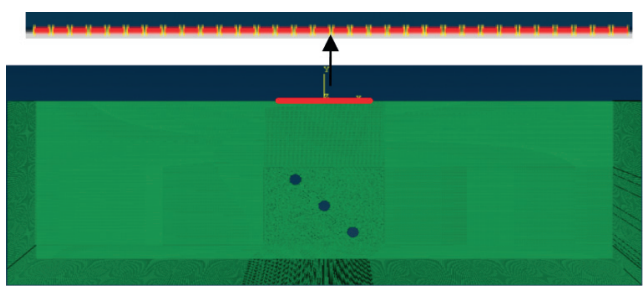

(a)

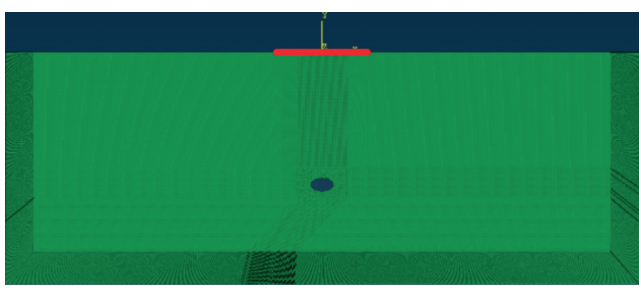

(b)

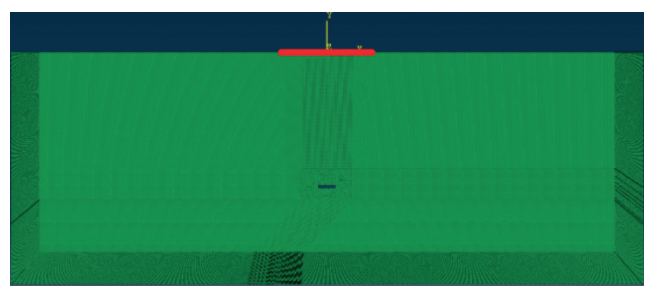

(c)

Figure 3: Finite element inspection model (the enlarged part is array chip): (a) round through-hole; (b) oval through-hole; (c) rectangular through-hole.

TABLE 1: Experimental array parameters.

\begin{tabular}{lc}
\hline Parameter & Value \\
\hline Number of elements & 32 \\
Element width & $0.4 \mathrm{~mm}$ \\
Element pitch & $0.1 \mathrm{~mm}$ \\
Sampling time interval & $2 \mathrm{e}-8 \mathrm{~s}$ \\
Center frequency & $5 \mathrm{MHz}$ \\
Wave velocity & $5800 \mathrm{~m} / \mathrm{s}$ \\
\hline
\end{tabular}

distance between the defect and the upper edge of the steel plate model was $20 \mathrm{~mm}$.

In this experiment, multiple analysis steps were set. Each element was excited by a 4 -cycle sinusoidal signal modulated by a Hanning window in sequence at each analysis step. At the same time, every array element received ultrasonic signal. Finally, data for $32 \times 32$ full matrix were captured. The signals were processed on MATLAB R2016a (MathWorks, Natick, MA, USA).

\subsection{Results and Analysis}

3.2.1. Symmetry of Full-Matrix Data. Figure 5 presents the amplitude data for $32 \times 32$ full matrix of round throughhole, oval through-hole, and rectangular through-hole, respectively. It can be seen that the data for full matrix were captured to be symmetrical. The result indirectly shows that only the upper or lower triangle data can be used for focus imaging, that is, half-matrix focus imaging. Therefore, the 


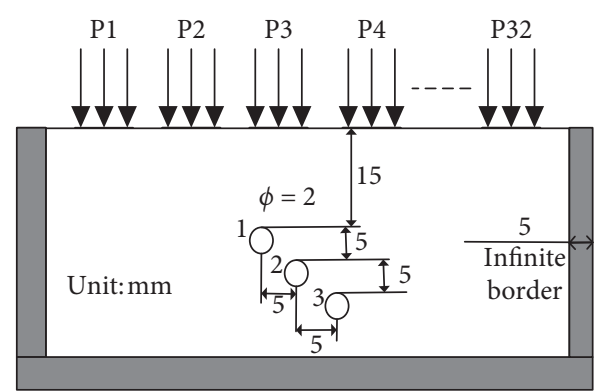

(a)

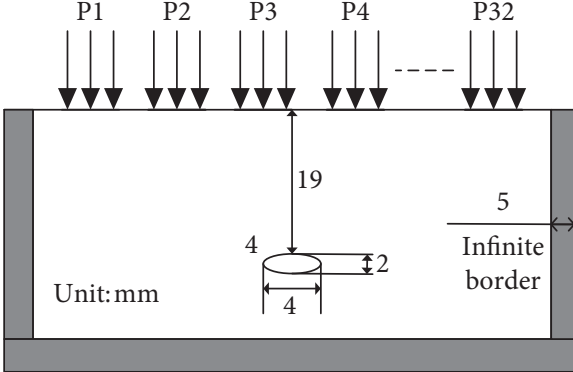

(b)

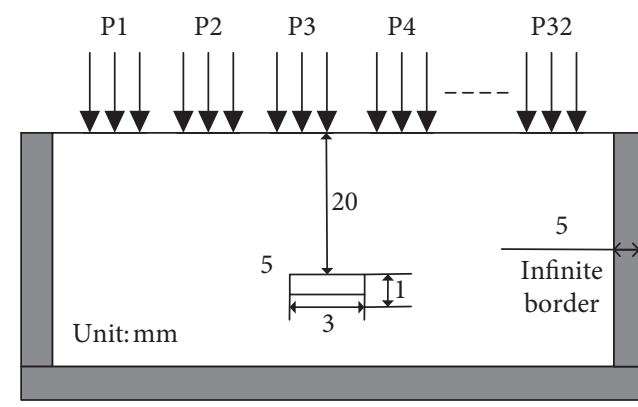

(c)

Figure 4: Schematic of defects in: (a) round through-hole; (b) oval through-hole; (c) rectangular through-hole.

resolution of half-matrix imaging is very close to that of fullmatrix focus imaging in theory.

3.2.2. Consistency of the Captured Signal. According to the reciprocity of the ultrasonic transceiver sensor, the acoustic signal captured by each transceiver channel remains consistent. The data of transceiver array elements exchange were extracted from full-matrix data. At the same time, the data were analyzed and compared. Here, six sets of A-scan data were randomly selected from full-matrix data. Signals received by different channels are shown in Figures 6-8. Figure 6 presents A-scan signal of defect Nos. 1, 2, and 3 detection. Figure 7 presents A-scan signal of defect No. 4 detection. Figure 8 presents A-scan signal of defect No. 5 detection. It can be seen that the transceiver element is exchanged, and the collected signal basically coincides. This fully demonstrates the reciprocity of the ultrasonic transceiver channel. This further illustrates that half-matrix data can be used in efficient focus imaging.

3.2.3. Comparison of Imaging Results. In the section, the difference between the imaging results of TFM and HFM was compared. According to the principle of TFM and HFM, we performed imaging experiments on round through-hole defects, oval through-hole defect, and rectangular throughhole defect, respectively. The imaging results are shown in Figures 9-11.
Comparing the two-dimensional imaging performance of the two algorithms, the five defects can be clearly displayed by TFM and HFM. However, it can be seen there is scattered noise around defects (mainly in the horizontal direction) by using the half-matrix imaging method. And, the shape and size of the defect have changed slightly. This is due to the small amount of data processed by the half-matrix imaging algorithm and poor signal average effect.

3.2.4. Image Synthesis A-Scan Signal and SNR. In order to quantify the imaging performance of the two methods, image synthesis A-scan signal is used as shown in Figure 12. It can be seen that image synthesis signals of Nos. 1-5 by TFM and HFM coincide. The amplitude and phase of the signal are basically equal.

For quantitatively analyzing the performance of the two imaging methods, the signal-to-noise ratio (SNR) is used to indicate the relationship between the defect signal and noise. The definition of SNR for a defect can be expressed [21-23] by

$$
\mathrm{SNR}=20 \times \log _{10}\left(\frac{A_{\max }}{A_{\text {ave }}}\right)
$$

where $A_{\max }$ is the maximum value (peak-to-peak) of the defect signal in the surrounding region and $A_{\text {ave }}$ is the 


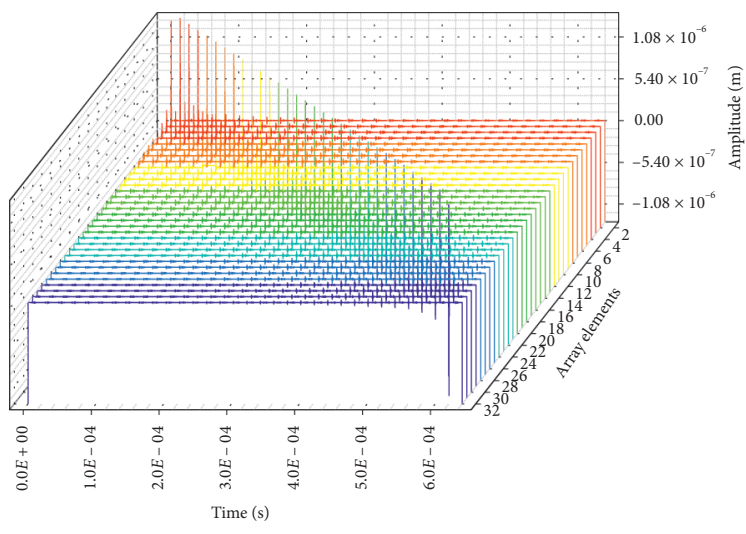

(a)

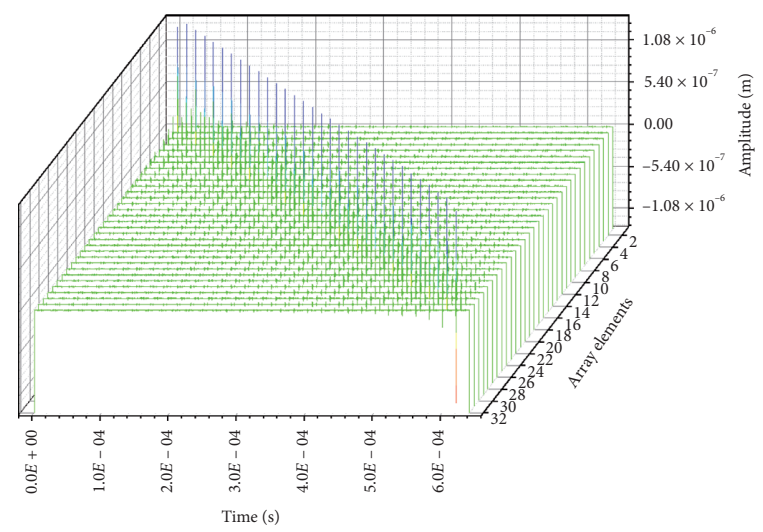

(b)

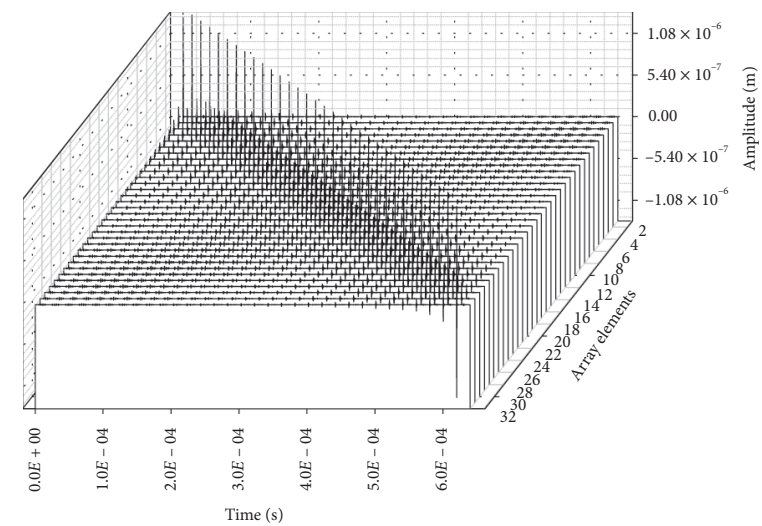

(c)

FIgURE 5: Full-matrix data: (a) round through-hole; (b) oval through-hole; (c) rectangular through-hole.

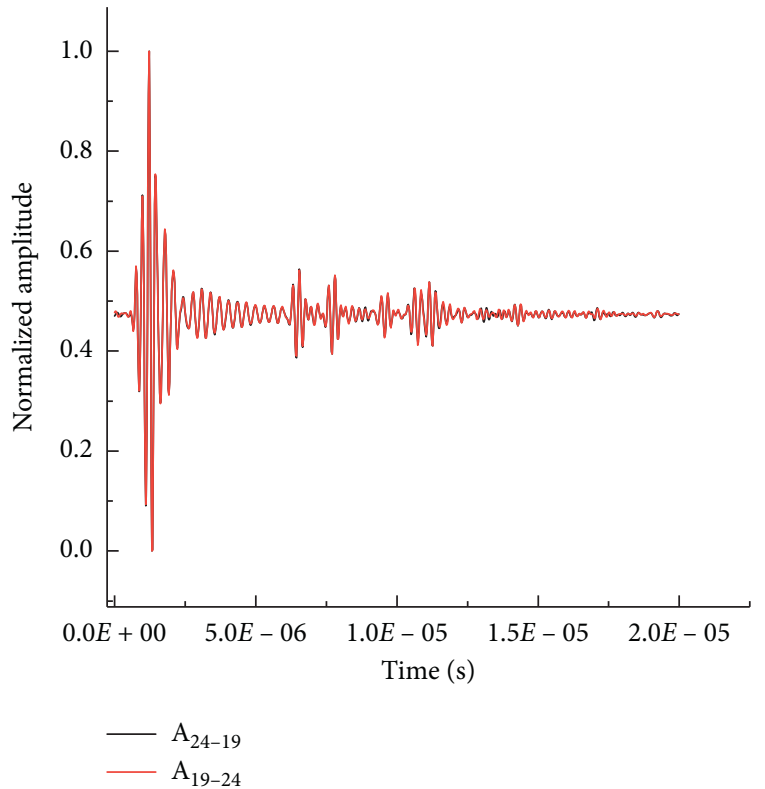

(a)

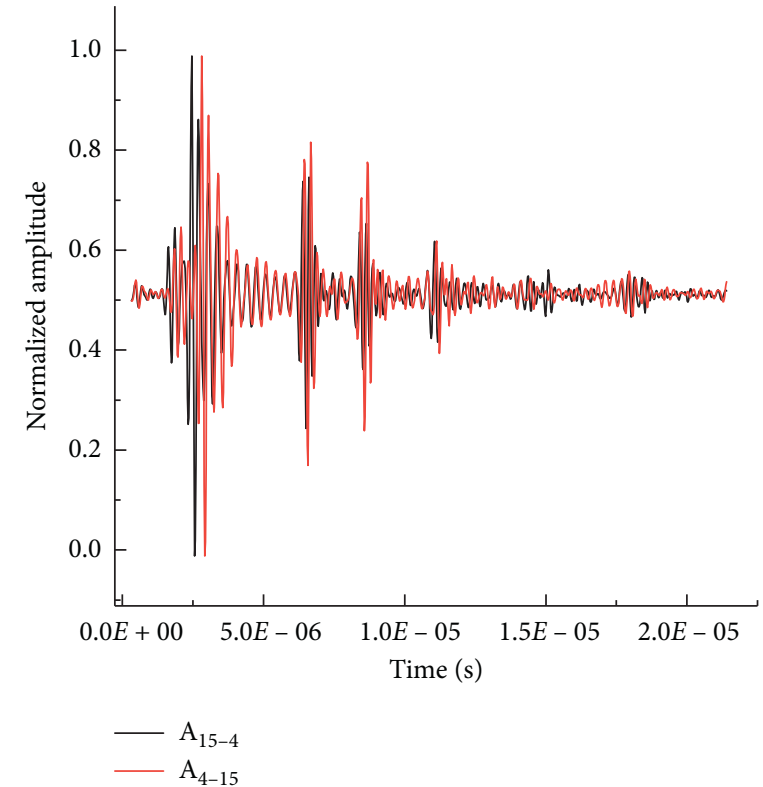

(b)

Figure 6: A-scan data of round through-hole: (a) $A_{24-19}$ and $A_{19-24}$; (b) $A_{15-4}$ and $A_{4-15}$. 


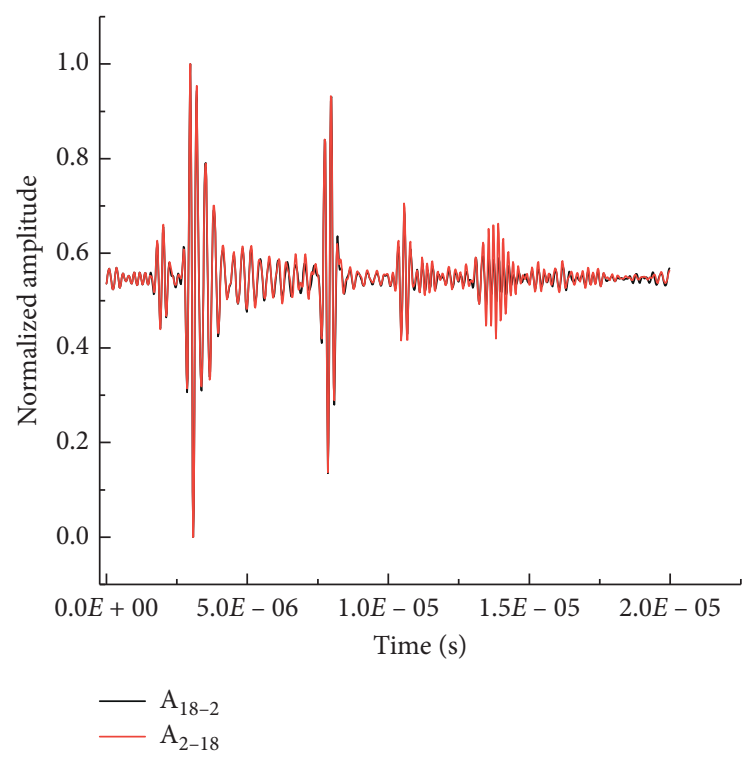

(a)

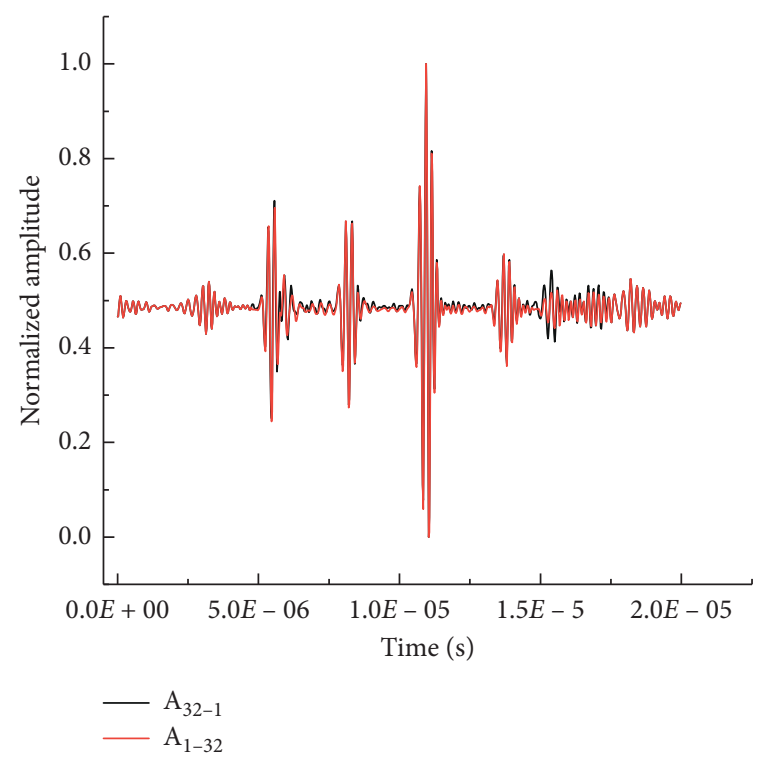

(b)

Figure 7: A-scan data of oval through-hole: (a) $A_{18-2}$ and $A_{2-18}$; (b) $A_{32-1}$ and $A_{1-32}$

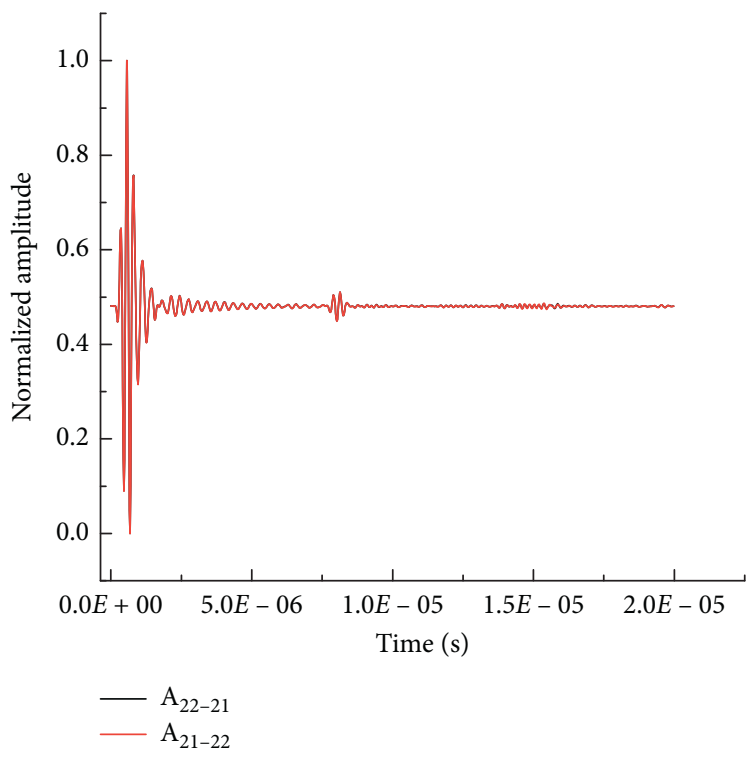

(a)

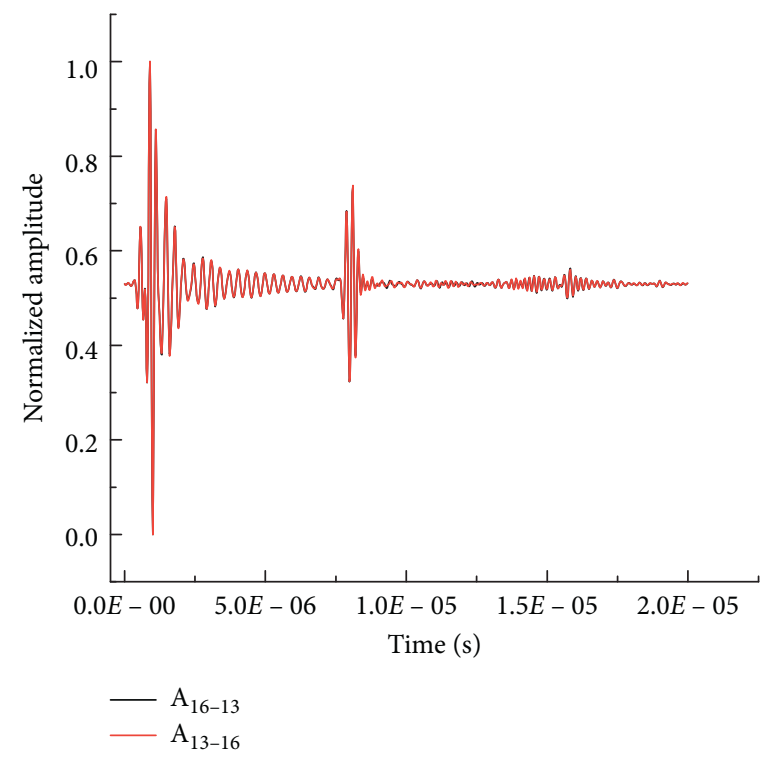

(b)

FIgURe 8: A-scan data of rectangular through-hole: (a) $A_{22-21}$ and $A_{21-22}$; (b) $A_{16-13}$ and $A_{13-16}$.

average of noise signal. Except for the defect signal, the rest of the data is regarded as a noise signal. Here, we analyzed the SNR of five defects in the three steel plate models for TFM and HFM. The results are shown in Figure 13.

The SNR for different defects by using TFM is better than the SNR by using HFM. But, the range of difference in SNR of TFM and HFM is very small. It can be seen that the difference range is between $1.09 \mathrm{~dB}$ and $1.87 \mathrm{~dB}$. Since more data are superimposed during total focus imaging, its waveform average effect is more obvious and the SNR is slightly higher.

In terms of imaging calculation time, the conditions are that the imaging pixel is set to $0.1 \mathrm{~mm} \times 0.1 \mathrm{~mm}$ and the imaging point is set to 1024000 . The CPU model of computer is Intel Core i5-9300H $(2.4 \mathrm{GHz})$, and RAM is $8 \mathrm{~GB}$. The full-matrix focus imaging takes 8.35 seconds, while the half-matrix focus 


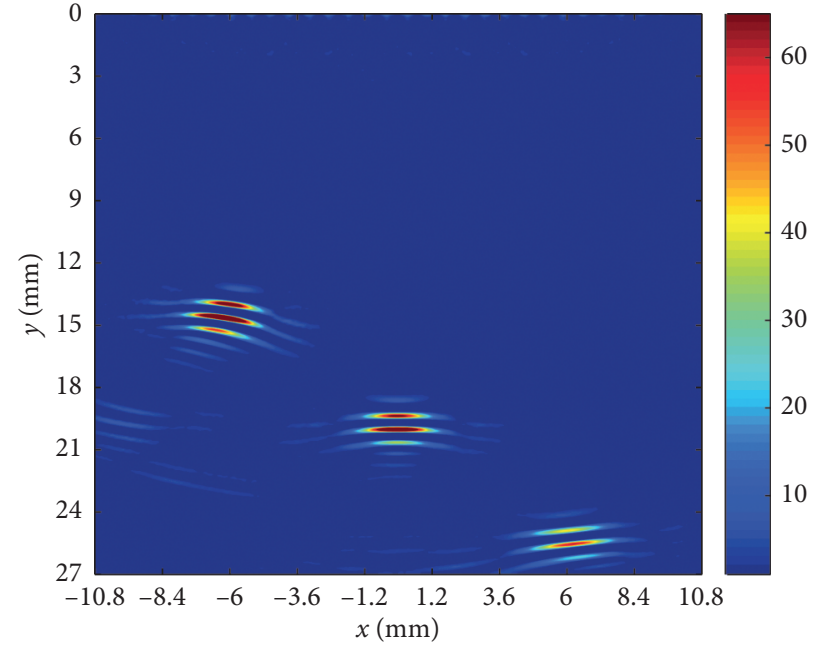

(a)

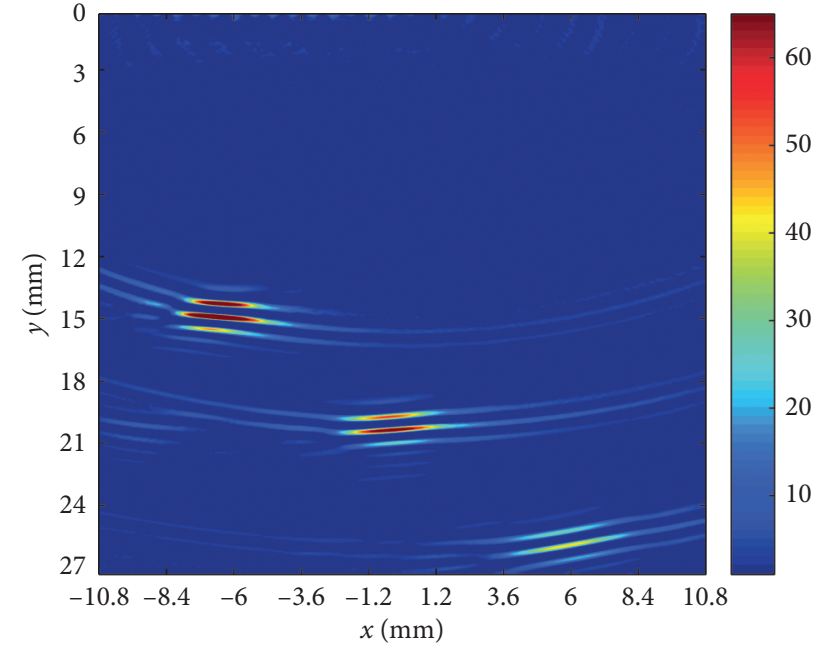

(b)

FIGURE 9: Focus imaging of round through-hole: (a) TFM; (b) HFM.

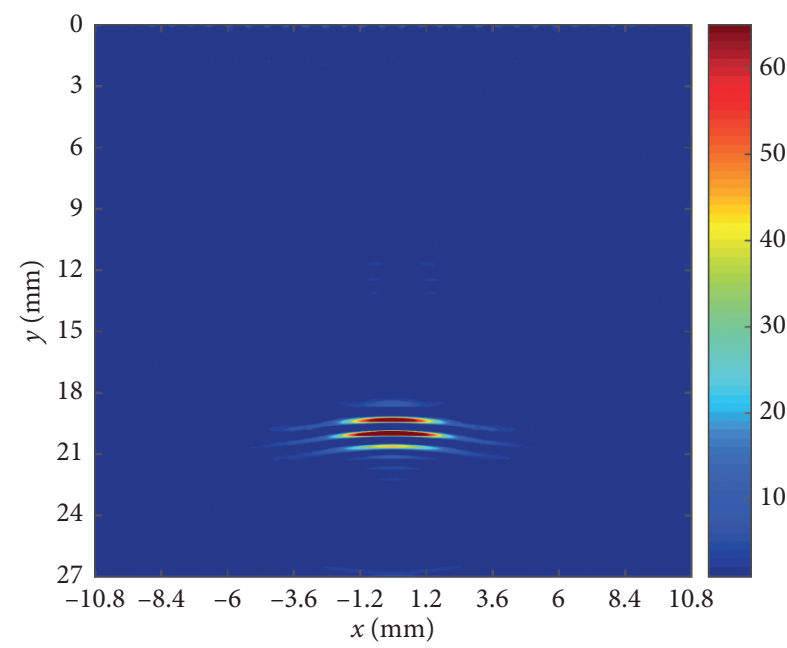

(a)

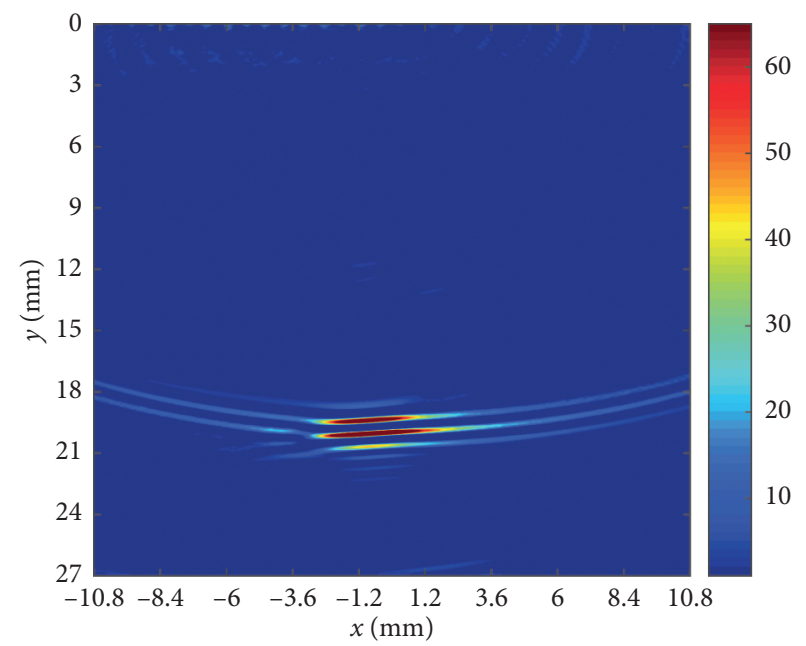

(b)

Figure 10: Focus imaging of oval through-hole: (a) TFM; (b) HFM.

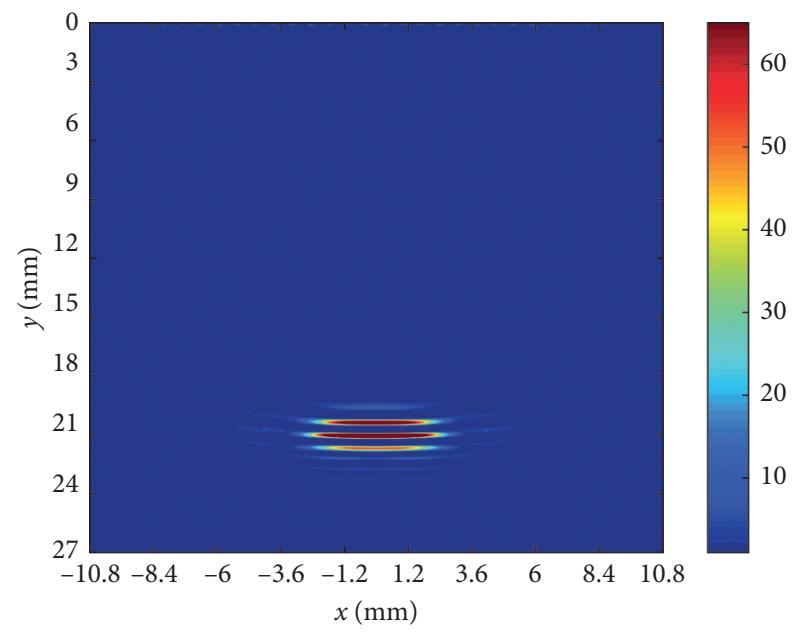

(a)

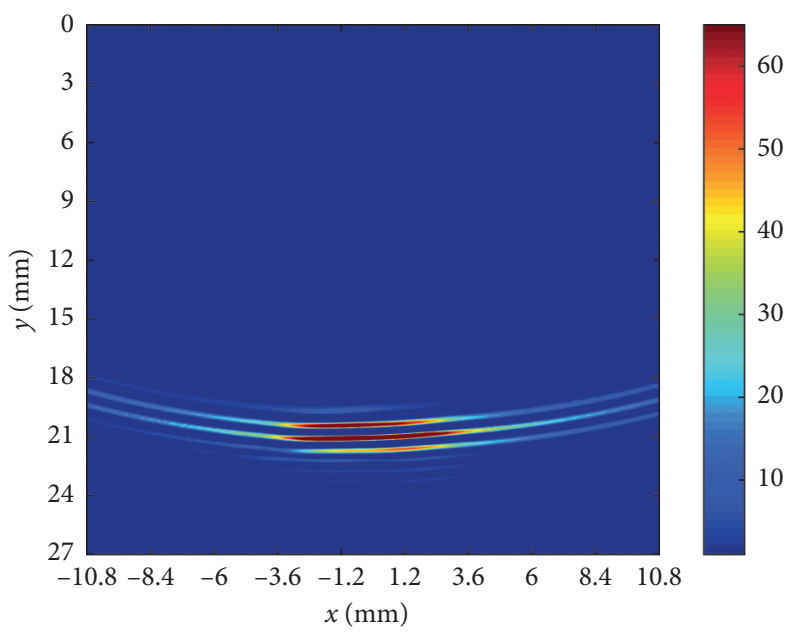

(b)

Figure 11: Focus imaging of rectangular through-hole: (a) TFM; (b) HFM. 


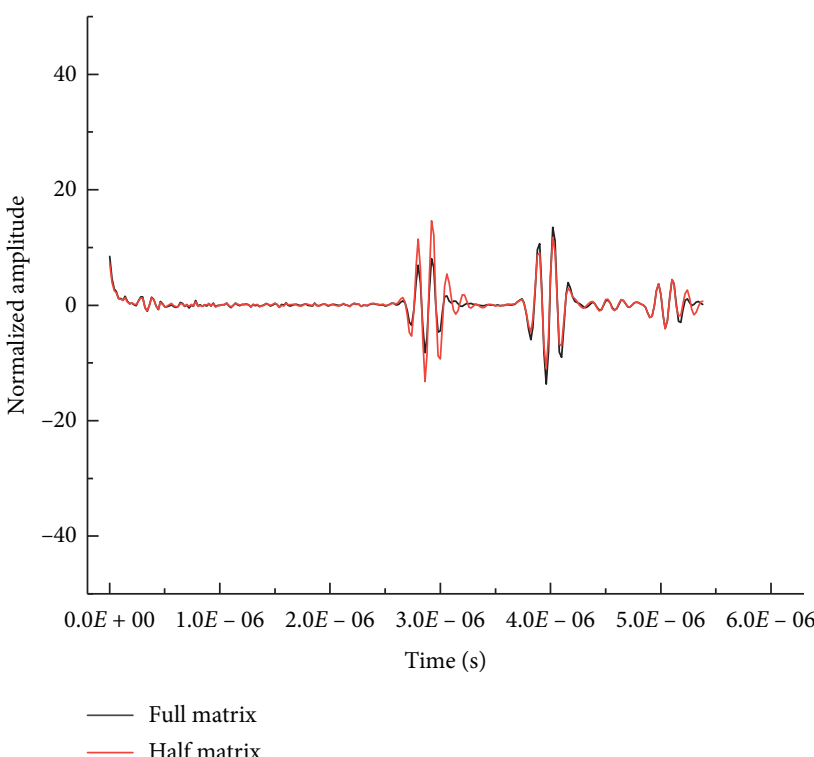

(a)

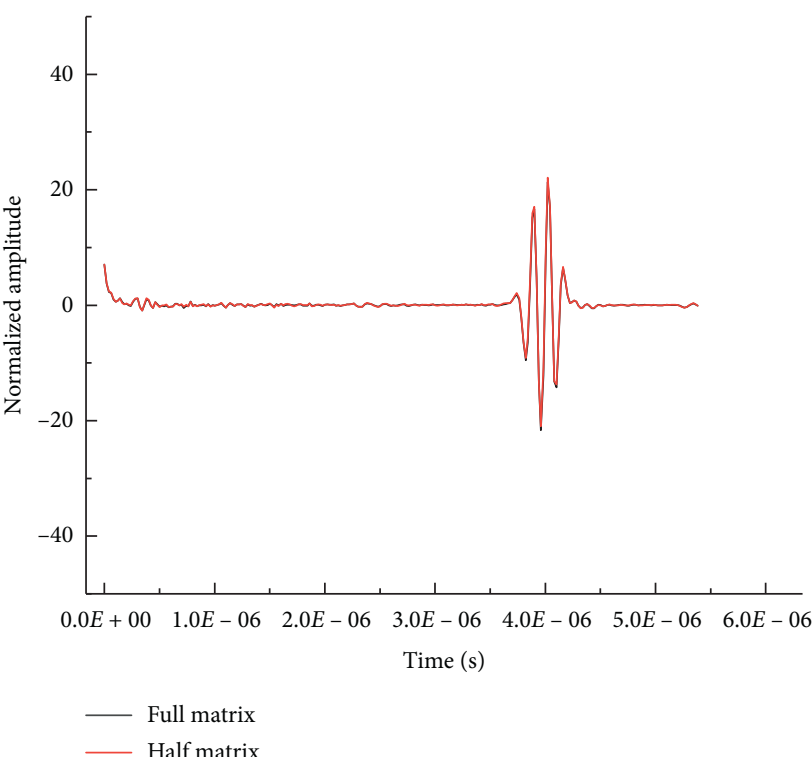

(b)

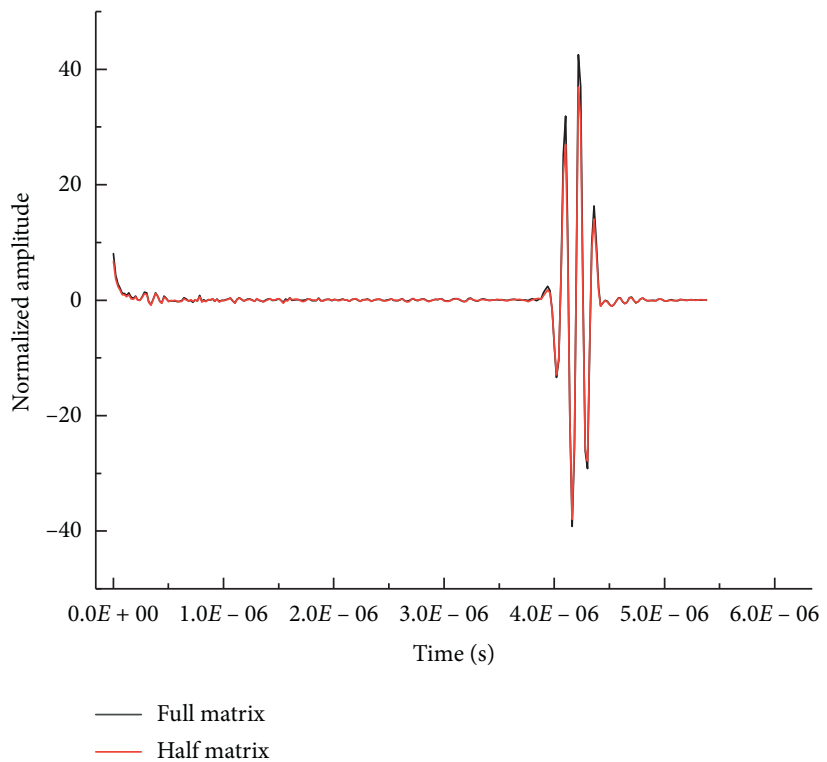

(c)

FIgURE 12: Image synthesis amplitude signal of defects: (a) round through-hole; (b) oval through-hole; (c) rectangular through-hole.

imaging takes 4.1 seconds. Compared with TFM, the imaging time by using HFM is approximately doubled and imaging efficiency greatly improved.

\section{Discussion}

The method used in the article is similar to that described in literature [21], but the experimental results are different. It can be seen from the experimental results that, compared with the TFM, the disadvantage of the HFM is its low signal-tonoise ratio. However, compared with traditional ultrasound B-scan imaging, HFM imaging has a higher signal-to-noise ratio. In the article, under the same experimental conditions,
B-scan imaging is performed on three kinds of defects, and the image obtained is shown in Figure 14. It can be seen that there is background noise in the image.

In the literature $[24,25]$, a microseismic waveform extraction method using unsupervised machine learning technique is proposed to improve the resolution of $\mathrm{mi}-$ croseismic imaging. With reference to the method of seismic imaging, machine learning and waveform feature extraction methods can also be used in ultrasound halfmatrix imaging to increase the signal-to-noise ratio and improve imaging resolution. For imaging efficiency, the literature [13] mentioned the use of GPU parallel acceleration to reduce the time of ultrasound TFM imaging. 


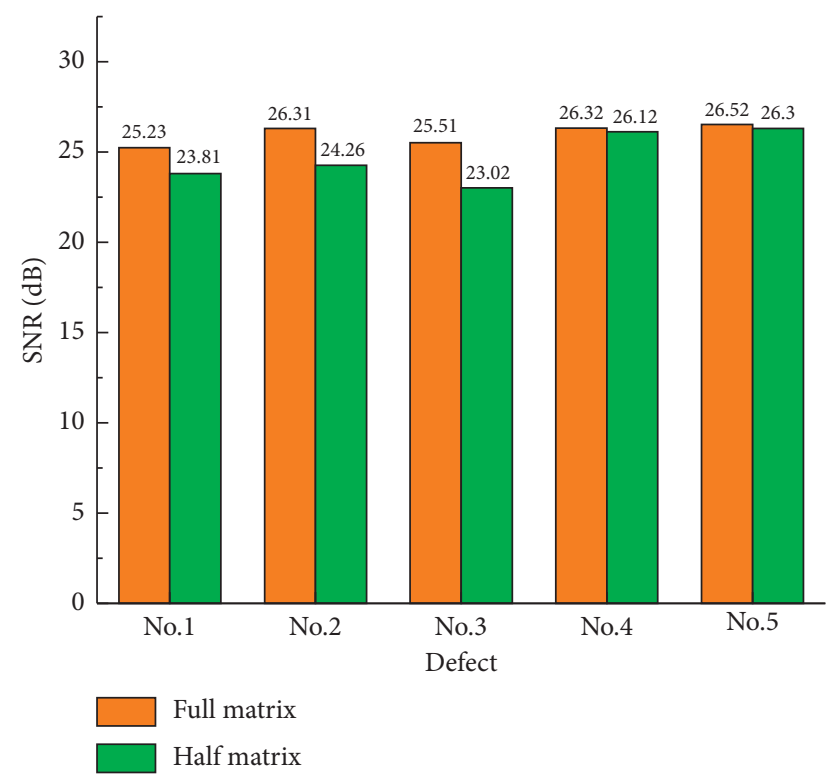

Figure 13: Comparison of signal-to-noise ratio of TFM and HFM.

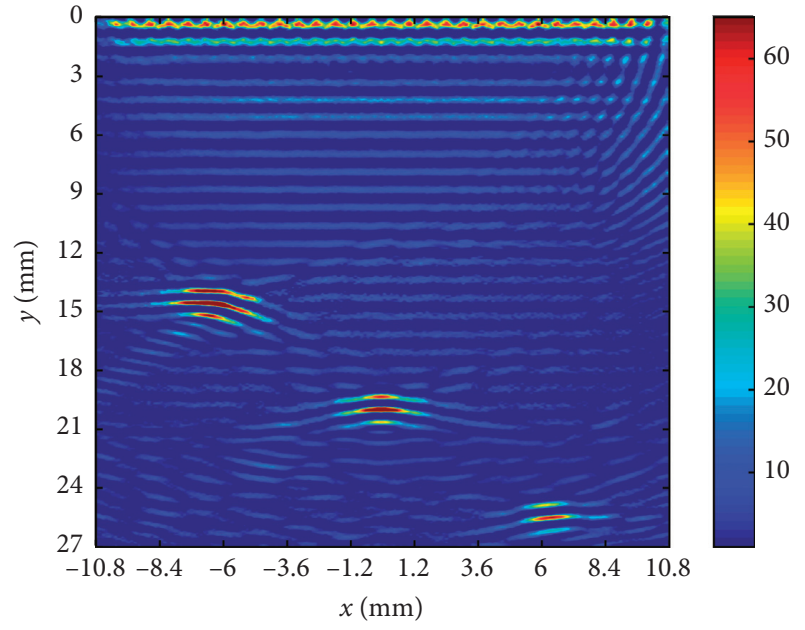

(a)

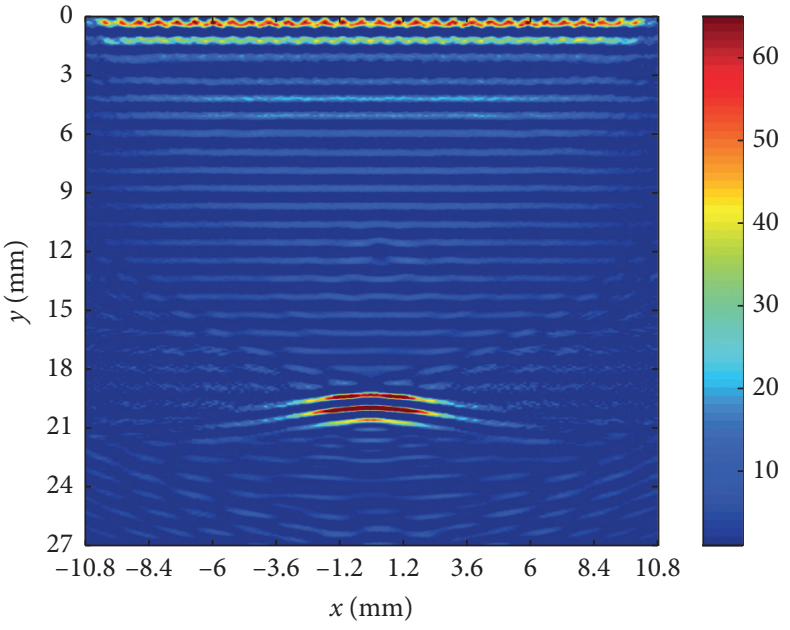

(b)

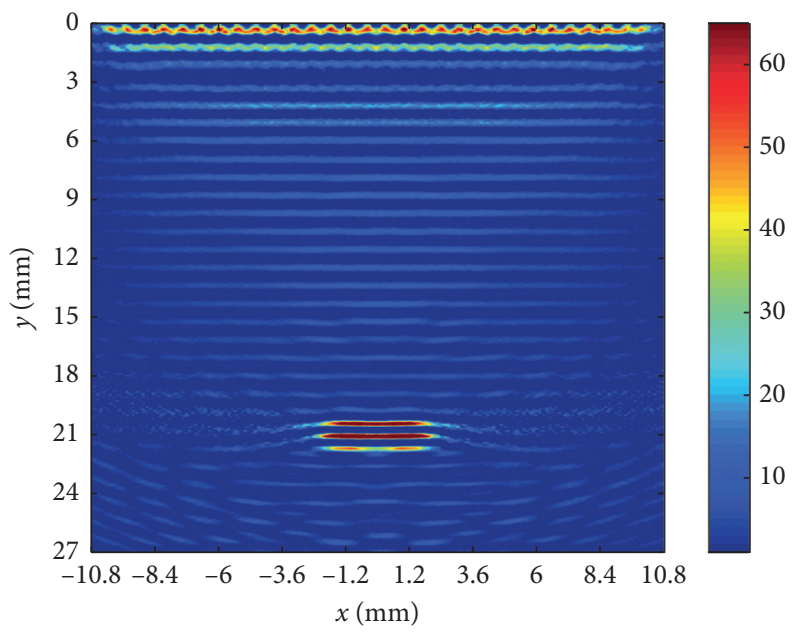

(c)

FiguRE 14: B-scan imaging of 3 different defects: (a) round through-hole; (b) oval through-hole; (c) rectangular through-hole. 
Therefore, the ultrasound HFM imaging can be combined with GPU parallel computing to further reduce the imaging time. The two methods discussed above deserve further research.

\section{Conclusions}

In this paper, a half-matrix focusing method based on transceiver sensor reciprocity is developed to focus imaging. The imaging quality and computational efficiency are analyzed. In terms of image performance, the synthesized signals of the half-matrix focus imaging algorithm and the full-matrix focus imaging algorithm have good consistency. But, the imaging resolution and SNR of TFM are slightly higher than those of HFM. However, the HFM can simplify the data acquisition and imaging operation process of the full-matrix focusing algorithm. The imaging time was approximately doubled. The HFM provide an important reference for the industrial application.

\section{Data Availability}

The data used to support the findings of this study are available from the corresponding author upon reasonable request.

\section{Disclosure}

The funders had role in the writing of the manuscript and in the decision to publish the results.

\section{Conflicts of Interest}

The authors declare no conflicts of interest.

\section{Acknowledgments}

The authors thank Prof. Han for his academic supervision and personal support. This work was supported by the Emergency Management Project of Natural Science Foundation of China (Grant no. 61842103) and Natural Science Foundation of Shanxi, China (Grant nos. 201801D121156 and 201901D111165).

\section{References}

[1] C. Li, D. Pain, P. D. Wilcox, and B. W. Drinkwater, "Imaging composite material using ultrasonic arrays," NDT \& E International, vol. 53, pp. 8-17, 2013.

[2] C. Yuan, C. Xie, L. Li, F. Zhang, and S. M. Gubanski, "Ultrasonic phased array detection of internal defects in composite insulators," IEEE Transactions on Dielectrics and Electrical Insulation, vol. 23, no. 1, pp. 525-531, 2016.

[3] X. X. Zhao, T. Gang, and B. X. Zhang, "Prediction of radiation beam fields from an array transducer with non-paraxial multiGaussian beam model," Artificial Intelligence in Federal Administrative Agencies, vol. 33, pp. 475-480, 2008.

[4] H. J. Hu, X. Zhu, C. H. Wang et al., "Stretchable ultrasonic transducer array three dimensional imaging on complex surfaces," Science Advances, vol. 4, pp. 1-11, 2018.
[5] C. Holmes, B. W. Drinkwater, and P. D. Wilcox, "Postprocessing of the full matrix of ultrasonic transmit-receive array data for non-destructive evaluation," NDT \& E International, vol. 38, no. 8, pp. 701-711, 2005.

[6] J. Zhang, B. W. Drinkwater, and P. D. Wilcox, "Effects of array transducer inconsistencies on total focusing method imaging performance," NDT \& E International, vol. 44, no. 4, pp. 361-368, 2011.

[7] J. Verkooijen and A. Boulavinov, "Sampling phased array a new technique for ultrasonic signal processing and imaging," Insight - Non-Destructive Testing and Condition Monitoring, vol. 50, pp. 153-157, 2007.

[8] A. J. Hunter, B. W. Drinkwater, and P. D. Wilcox, "Autofocusing ultrasonic imagery for non-destructive testing and evaluation of specimens with complicated geometries," NDT \& E International, vol. 43, no. 2, pp. 78-85, 2010.

[9] A. Velichko and P. D. Wilcox, "Reversible back-propagation imaging algorithm for postprocessing of ultrasonic array data," IEEE Transactions on Ultrasonics, Ferroelectrics and Frequency Control, vol. 56, no. 11, pp. 2492-2503, 2009.

[10] C. J. L. Lane, T. K. Dunhill, B. W. Drinkwater, and P. D. Wilcox, "3D ultrasonic inspection of anisotropic aerospace components," Insight - Non-destructive Testing and Condition Monitoring, vol. 52, no. 2, pp. 72-77, 2010.

[11] J. Russell, R. Long, D. Duxbury, and P. Cawley, “Development and implementation of a membrane-coupled conformable array transducer for use in the nuclear industry," Insight Non-destructive Testing and Condition Monitoring, vol. 54, no. 7, pp. 386-393, 2012.

[12] E. Moghimirad, C. A. Villagomez Hoyos, A. Mahloojifar, B. Mohammadzadeh Asl, and J. A. Jensen, "Synthetic aperture ultrasound Fourier beamformation using virtual sources," IEEE Transactions on Ultrasonics, Ferroelectrics, and Frequency Control, vol. 63, no. 12, pp. 2018-2030, 2016.

[13] M. Sutcliffe, M. Weston, B. Dutton, P. Charlton, and K. Donne, "Real-time full matrix capture for ultrasonic nondestructive testing with acceleration of post-processing through graphic hardware," NDT \& E International, vol. 51, pp. 16-23, 2012.

[14] M. Njiki, A. Elouardi, S. Bouaziz, O. Casula, and O. Roy, “A multi-FPGA architecture-based real-time TFM ultrasound imaging," Journal of Real-Time Image Processing, vol. 16, no. 2, pp. 505-521, 2019.

[15] S. Bouaziz, M. Njiki, A. Elouardi, O. Casula, and O. Roy, “A real-time implementation of the Total Focusing Method for rapid and precise diagnostic in nondestructive evaluation," in Proceedings of the IEEE 24th International Conference on Application Specific Systems, pp. 245-248, Architectures and Processors, Washington, DC, USA, June 2013.

[16] A. J. Hunter, B. W. Drinkwater, and P. D. Wilcox, "The wavenumber algorithm for full-matrix imaging using an ultrasonic array," IEEE Transactions on Ultrasonics, Ferroelectrics and Frequency Control, vol. 55, no. 11, pp. 2450-2462, 2008.

[17] H. Hu, J. Du, C. Ye, and X. Li, "Ultrasonic phased array sparse-TFM imaging based on sparse array optimization and new edge-directed interpolation," Sensors, vol. 18, no. 6, p. 1830, 2018.

[18] L. Moreau, B. W. Drinkwater, and P. D. Wilcox, "Ultrasonic imaging algorithms with limited transmission cycles for rapid nondestructive evaluation," IEEE Transactions on Ultrasonics, Ferroelectrics and Frequency Control, vol. 56, no. 9, pp. 1932-1944, 2009. 
[19] J. N. Potter, P. D. Wilcox, and A. J. Croxford, "Diffuse field full matrix capture for near surface ultrasonic imaging," Ultrasonics, vol. 82, pp. 44-48, 2018.

[20] H. Zhang, Y. Liu, G. Fan, H. Zhang, W. Zhu, and Q. Zhu, "Sparse-TFM imaging of lamb waves for the near-distance defects in plate-like structures," Metals, vol. 9, no. 5, p. 503, 2019.

[21] X. Zhao, T. Z. Qi, Z. Y. Wang et al., "Ultrasonic detection triangle matrix focus imaging algorithm," Journal of $\mathrm{Me}$ chanical Engineering, vol. 55, no. 4, pp. 19-24, 2019.

[22] H. Zhang, L. Zeng, G. Fan, H. Zhang, Q. Zhu, and W. Zhu, "Instantaneous phase coherence imaging for near-field defects by ultrasonic phased array inspection," Sensors, vol. 20, no. 3, p. $775,2020$.

[23] A. Velichko and P. D. Wilcox, "An analytical comparison of ultrasonic array imaging algorithms," The Journal of the Acoustical Society of America, vol. 127, no. 4, pp. 2377-2384, 2010.

[24] Y. Chen, "Fast waveform detection for microseismic imaging using unsupervised machine learning," Geophysical Journal International, vol. 215, no. 2, pp. 1185-1199, 2018.

[25] M. S. Omar and Y. K. Chen, "Automatic waveform-based source-location imaging using deep learning extracted microseismic signals," Geophysics, vol. 85, pp. 171-183, 2020. 